\title{
Clinical Features of COVID-19 in a Young Man with Massive Cerebral Hemorrhage-Case Report
}

\author{
Yi Bao $^{1}$ (D) Shu Yu Lin ${ }^{2} \cdot$ Zhao Hui Cheng $^{1} \cdot$ Jun Xia ${ }^{2} \cdot$ Yan Peng Sun ${ }^{1} \cdot$ Qi Zhao $^{1} \cdot$ Guang Jian Liu ${ }^{1}$
}

Accepted: 11 May 2020/Published online: 23 May 2020

(C) Springer Nature Switzerland AG 2020

\begin{abstract}
COVID-19 is currently a pandemic in the world, can invade multiple systems, and has a high morbidity and mortality. So far, no cases of acute cerebrovascular disease have been reported. This article reports the clinical features of a COVID-19 patient whose first symptom was cerebral hemorrhage. More importantly, after the craniotomy, the patient had high fever and it was difficult to retreat. After cerebrospinal fluid testing, it was determined that an intracranial infection had occurred. After anti-infection and plasma infusion of the recovered person, the patient's symptoms gradually improved. This case suggests that COVID-19 may infringe on cerebral blood vessels and cause cerebral hemorrhage. Transfusion of plasma from rehabilitation patients is effective for critically ill patients.
\end{abstract}

Keywords COVID-19 · Cerebral hemorrhage $\cdot$ SARS-CoV-2 $\cdot$ ACE2 $\cdot$ Immunoglobulin

\section{Introduction}

Since December 2019, COVID-19 was first discovered in Wuhan, China [1]. At present, the disease has occurred in a worldwide pandemic, is highly contagious, has a high morbidity and mortality, and has been declared a public health emergency of international concern by the World Health Organization (WHO) [2-4]. As of April 10, it has caused a total of 1.6 million patients, of which more than 95,000 died. The most typical symptom of COVID-19 is respiratory distress. More and more evidence shows that SARS-CoV-2 is not always limited to the respiratory system, but can also violate the cardiovascular, digestive tract, kidney, nervous system, and reproductive systems [5-7]; symptoms of nervous system are divided into three categories: central nervous system (CNS) symptoms (headache, dizziness, acute cerebrovascular disease, ataxia, disturbance of consciousness, epilepsy), and

This article is part of the Topical Collection on Covid-19

Yi Bao

karlbaoyi@163.com

1 Department of Neurology, Taihe Hospital Affiliated to Hubei University of Medicine, Shiyan City, Hubei Province, China

2 Intensive Care Unit (ICU), Taihe Hospital Affiliated to Hubei University of Medicine, Shiyan City, Hubei Province, China peripheral nervous system (PNS) symptoms (hyposmia, taste loss, loss of appetite, neuralgia), skeletal muscle damage [8-13]. However, no cases related to acute cerebrovascular disease have been reported so far. This article reports a case of COVID-19 complicated with cerebral hemorrhage and summarizes the relevant treatment experience, which is now shared as follows.

\section{Case Reports}

At 19:00 on February 16, 2020, a 38-year-old man suffered a sudden disturbance of consciousness during dinner and fell to the ground. He could not be awakened, could not speak, accompanied by vomiting, no abnormal limb movements and convulsions, and no incontinence. After onset, the family sent him to the local town health center. After symptomatic treatment, the patient's symptoms did not get better, so the patient was transferred to the local people's hospital for emergency treatment. Computed tomography (CT) examination of the brain revealed a large amount of cerebral hemorrhage, and the family was informed that the patient was in critical condition (Fig. 1).

At 15:00 on February 17, the patient presented with difficulty breathing and poor blood oxygen saturation. After endotracheal intubation, breathing was assisted by ventilator. For further treatment, the patient was transferred to the 


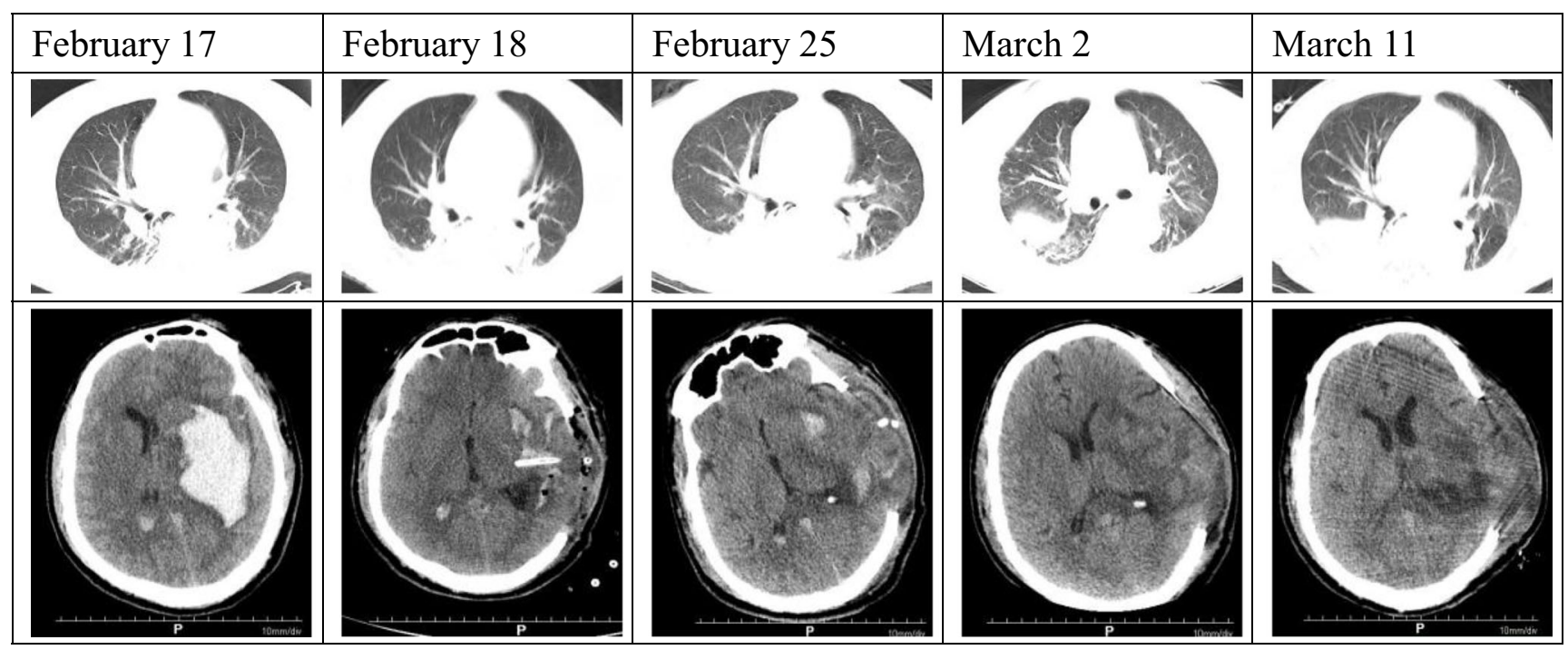

Fig. 1 CT changes of lung and brain in patients with COVID-19 complicated with cerebral hemorrhage

cerebrovascular disease treatment center of our hospital at 21:00 pm.

The patient's past history was obtained by asking family members: physical fitness, denying the history of hypertension, diabetes, and heart disease; denying a history of hepatitis $\mathrm{B}$ and tuberculosis; denying a history of food and drug allergies; and denying a history of trauma and surgery. Contact history of the epidemic area and close contact history of COVID-19 confirmed patients were denied. Physical examination: T $36.8^{\circ} \mathrm{C}$, P 65 times/min, R 15 times/min, BP 155/ $106 \mathrm{mmHg}$, acute physiology and chronic health evaluation scoring systemII (APACHE II) scoring 16 points, conscious coma, endotracheal intubation, indwelling urinary catheter, examination cannot cooperate. There was no trauma or deformity in the skull, the pupil was unequal on both sides, the diameter of the left side was $3.0 \mathrm{~mm}$, the diameter of the right side was $2 \mathrm{~mm}$, and the light reflection was weakened. The neck was soft, the breath sounds in both lungs were rough, moist rales could be heard at the bottom of the lungs, the heart rhythm was uniform, no obvious murmurs were heard in the auscultation area of each valve of the heart, the abdomen was soft, and the liver and spleen were not touched. There was flexion in the left limb tingling, muscle strength level 3, poor response in the right limb tingling, muscle strength level 1 , increased muscle tension, and the right Barbinski sign was positive.

Examination data showed: white blood cells (WBC) $13.19 \times 10^{9} \mathrm{G} / \mathrm{L}$, neutrophil absolute value (NE\#) $12.06 \times 10^{9} \mathrm{G} / \mathrm{L}$, neutrophil percentage (NE\%) $91.5 \%$, lymphocyte absolute value (LY\#) $0.39 \times 10^{9} \mathrm{G} / \mathrm{L}$, lymphocyte percentage (LY\%) $2.9 \%$, and high sensitivity C-reactive protein (hsCRP) $70.7 \mathrm{mg} / \mathrm{L}$. Emergency cranial CT examination showed that there were large irregular high-density lesions in the left temporal lobe, basal ganglia, and radiating coronal area, with uniform density and clear edges, the maximum layer size was about $8.5 \times 4.2 \mathrm{~cm}$, there were large patches of low-density edema around, the left ventricle was compressed and deformed, and the midline structure was shifted to the right; high-density shadows were seen in the bilateral ventricles. Conclusion: the left temporal lobe, basal ganglia, and radiation coronal hematomas break into the ventricles and brain hernias were formed. Chest CT results showed that there were cords and strip-shaped high-density shadows on the upper and lower lobe of both lungs, and the state was blurred; no abnormal density shadows were found in the remaining lungs. Endotracheal intubation, clear hilar structure, no displacement of the mediastinum, no obvious enlarged lymph nodes, no pleural effusion. Conclusion: sedimentary inflammation in both lungs.

At 23:00 on February 17, in order to remove the intracranial hematoma, after informed consent and signature by the patient's family, the operation was performed under general anesthesia: removal of left intracerebral hematoma + decompression of the left cranial flap + dura meningiotomy. During the operation, a dark red blood clot was seen in the left basal ganglia, and the clot was removed. No aneurysm and vascular malformation were seen during the operation. One drainage tube was placed in the hematoma cavity. After removing the bone flap, a second drainage tube was placed outside the dura mater, and the muscle, subcutaneous, and scalp were sutured, and the drainage tube was fixed. Safe transfer to intensive care unit (ICU) after surgery. Continue to use ventilator to assist breathing, oxygen inhalation, electrocardiogram (ECG) monitoring, anti-infection (Flucloxacillin), hemostasis (Aminocaproic acid), dehydration (Mannitol), brain protection, prevention of epilepsy, and other treatments, as well as symptomatic support treatment, and closely observed the changes of consciousness and pupil (Fig. 2). 
Fig. 2 The treatment of COVID19 patients with intracerebral hemorrhage

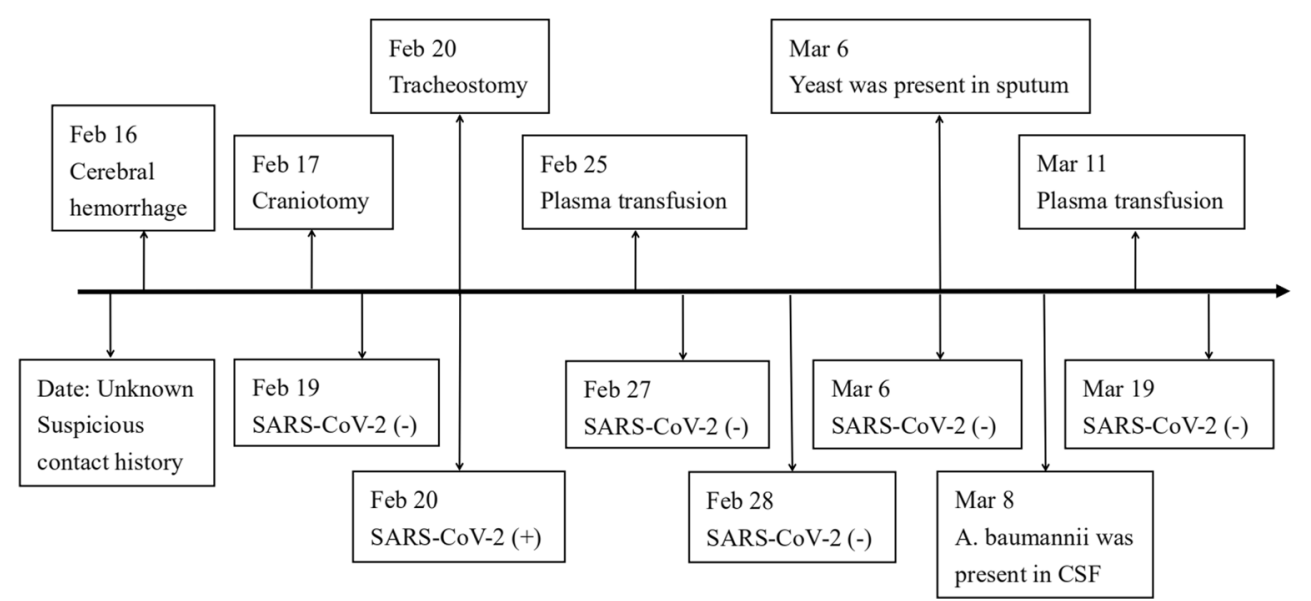

On February 18, the patient had a high fever with a body temperature of $39.6^{\circ} \mathrm{C}$, Re-examination of the craniocerebral CT revealed that: post craniocerebral operation, intracranial gas was accumulated, a drainage tube was placed, part of the left frontal parietal temporal bone was missing, and the left temporal lobe, basal ganglia, and radiative coronal area showed patchy high-density lesions, which were significantly reduced compared with the previous (February 17, 2020). The center line structure was less offset to the right than before; high-density shadows on both sides of the ventricle were slightly reduced compared with before. The scalp soft tissue was swollen and gas was accumulated. Re-examination of the chest $\mathrm{CT}$ showed that there were patches and strip-like highdensity shadows on the back of both lungs, with a blurred state and partial consolidation, which was more than before (February 17, 2020). Considering the aggravation of lung infection, meropenem was used to continue anti-infection treatment.

On February 19, the patient still had high fever, excessive phlegm, and low blood oxygen saturation. Rough breath sounds could be heard at auscultation, and both lung breath sounds were low. Asked the family members of the patient to know that the close contact history of COVID-19 could not be completely ruled out. COVID-19 should be considered in combination with the patient's chest CT, which showed pulmonary interstitial inflammation, blood routine lymphocytic reduction, and poor anti-bacterial treatment effect, etc. Then the patient samples (pharyngeal swabs, sputum, serum, and stool) were taken for real-time fluorescence quantitative reverse transcription-polymerase chain reaction (rRT-PCR), and SARS-CoV-2 antibody-IgG and IgM tests were completed; the results were negative.

On the 20th, the patient's blood oxygen saturation could not be maintained, dyspnea was aggravated, and sputum was difficult to be sucked out. Then, tracheostomy was performed under local anesthesia, and the ventilator was used to assist breathing. In order to further improve the positive rate, the reexamination of sputum results showed that the SARS-CoV-2 nucleic acid test was positive. The COVID-19 consultation expert group considered: combining epidemiology, lymphocytic decrease, increased CRP, pulmonary interstitial inflammation, and SARS-CoV-2 nucleic acid positive; COVID-19 could be confirmed. In treatment, Abidol combined with Ribavirin antiviral, Alpha-Interferon nebulization treatment, thymosin to enhance immunity, and continued anti-infective treatment. Nursing: paid attention to strengthening airway management, strengthening atomization, sucking sputum in time, and turning back and patting frequently.

On February 24, the patient developed high fever again with a body temperature of $38.8^{\circ} \mathrm{C}$ (Fig. 3). The cerebrospinal fluid became turbid as before. The examination showed that the cerebrospinal fluid (CSF) was dark red, no clot, protein was $2.5 \mathrm{~g} / \mathrm{L}$, sugar was $1.85 \mathrm{mmol} / \mathrm{L}$, and white blood cells were $673 \times 10^{6} \mathrm{G} / \mathrm{L}$, among which multinucleated cells accounted for $95 \%$ (Table 1). Cerebrospinal fluid test results showed intracranial infection; the first consideration was SARS-CoV-2 intracranial infection. Check the cerebrospinal fluid SARS-CoV-2 nucleic acid RNA, and the result was negative. Continue to give antiviral and antibacterial treatment.

On February 25, in order to alleviate the patient's condition, with the consent of the patient's family, $150 \mathrm{ml}$ plasma of type A Rh positive COVID-19-convalved patient was given. Re-examination of the brain CT showed that the scope of the patchy high-density foci in the left temporal lobe, basal ganglia, and radiating coronal region was approximately the same as before, the density was lower than before (2020-2-18), and the midline structure shifted to the right was less than before; the density of bilateral ventricles was slightly reduced. Reexamination of the chest $\mathrm{CT}$ results showed that there were patches and strip-shaped high-density shadows on the back of the two lungs, with a blurred state and partial consolidation, which was reduced from the previous (February 18, 2020).

On February 27, after the patient was infused with COVID19 convalescent plasma, the symptoms improved, the body temperature decreased, and the dyspnea decreased. Both SARS-CoV-2 nucleic acid tests were negative ( $24 \mathrm{~h}$ interval), 
Fig. 3 Temperature curve of COVID-19 patients with intracerebral hemorrhage

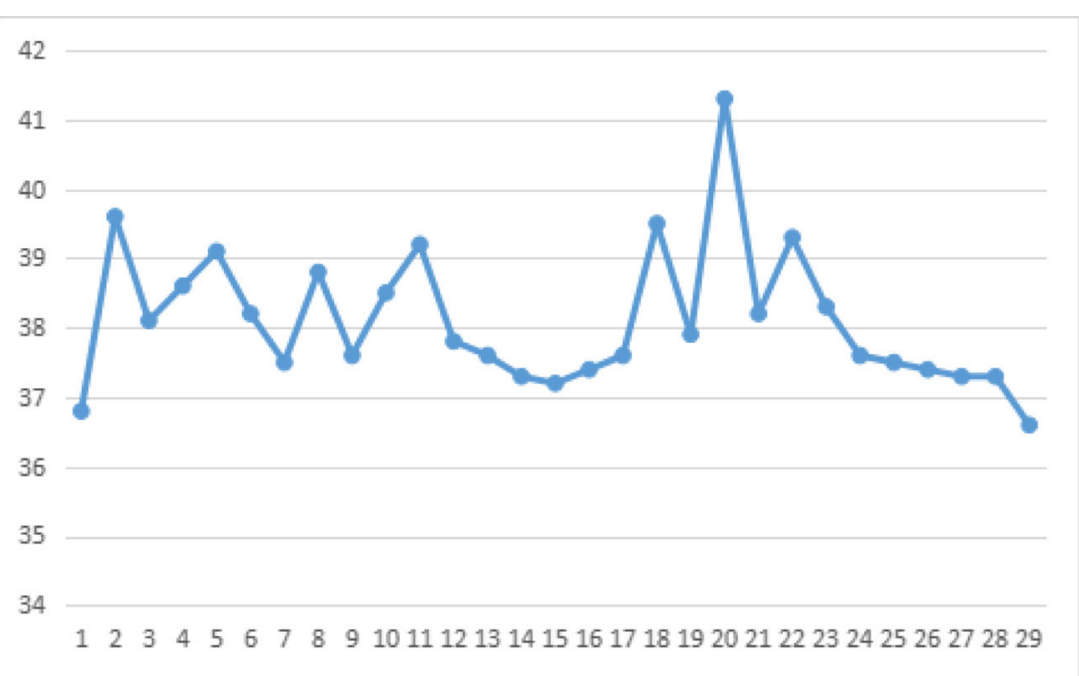

Y-coordinate: body temperature $\left({ }^{\circ} \mathrm{C}\right)$

$\mathrm{X}$-coordinate: time from February 17. suggesting that antiviral treatment was effective. However, in the afternoon of the same day, the patient developed hyperthermia again, and his body temperature reached $39.2^{\circ} \mathrm{C}$; considering the central hyperthermia, the patient improved after depyrexia treatment.

On February 29, the patient did not have high fever again, the results of the cerebrospinal fluid review showed that it was light red, no clot, protein decreased to $0.8 \mathrm{~g} / \mathrm{L}$, sugar increased to $4.45 \mathrm{mmol} / \mathrm{L}$, and white blood cells decreased to $37 \times$ $10^{6} \mathrm{G} / \mathrm{L}$, of which monocytes accounted for $74 \%$. This indicated that the intracranial infection was better than before.

On March 2, for several days, the patient's temperature was around $37.2^{\circ} \mathrm{C}$. A review of cranial CT showed that the range of patchy high-density lesions was narrower than before (2020-2-25), the left ventricle was compressed and deformed, the degree to which the centerline structure shifts to the right was lessened than before; high-density shadows in the ventricle were slightly reduced, the soft tissue swelling of the scalp was more obvious than before. Re-examination of the chest CT showed that there were patches and strip-like high-density shadows on the back of both lungs, with a blurred state and partial consolidation, which was more than before (February 25, 2020). Review of CSF: CSF was pink, no clots, protein decreased to $0.59 \mathrm{~g} / \mathrm{L}$, sugar $3.47 \mathrm{mmol} / \mathrm{L}$, slightly lower than before, white blood cells rose to $102 \times 10^{6} \mathrm{G} / \mathrm{L}$, mononuclear cells accounted for $60 \%$. The patient's cerebrospinal fluid showed improvement, and since the two re-examinations of SARS-CoV-2 nucleic acid test was negative, and the antiviral treatment with Abidol, Ribavirin, and Oseltamivir had reached the course of treatment, so it was discontinued.

On March 5, the patient developed hyperthermia again, the body temperature reached $39.5^{\circ} \mathrm{C}$, and the cerebrospinal fluid with clots was drained from the lumbar cistern. The test results showed that the cerebrospinal fluid was light red with clots, protein significantly increased to $4.28 \mathrm{~g} / \mathrm{L}$, sugar decreased to $0.01 \mathrm{mmol} / \mathrm{L}$, and white blood cells increased to $172 \times 10^{6} \mathrm{G} /$

Table 1 CSF changes in COVID-19 with intracerebral hemorrhage

\begin{tabular}{|c|c|c|c|c|c|c|c|}
\hline & Feb 24 & Feb 29 & Mar 02 & Mar 05 & Mar 07 & Mar 12 & Mar 14 \\
\hline $\operatorname{Protein}(\mathrm{g} / \mathrm{L})$ & 2.5 & 0.8 & 0.59 & 4.28 & 4.29 & 3.63 & 2.57 \\
\hline Chlorine(mmol/L) & 122.9 & 107 & 125.2 & 125.3 & 140.3 & 140 & 123.9 \\
\hline Glucose(mmol/L) & 1.85 & 4.45 & 3.47 & 0.01 & 0.4 & 1.69 & 1.41 \\
\hline HsCRP(mg/L) & 1.84 & 0.54 & 0.62 & 15.46 & 15.52 & 4.97 & 4.26 \\
\hline Coloer & Crimson & Light red & Light red & Light red & Yellow & Yellow & Light yellow \\
\hline Character & Turbid, no clot & Turbid, no clot & Turbid, no clot & Turbid, with clot & Turbid, with clot & Turbid, no clot & Turbid, no clot \\
\hline $\mathrm{WBC}\left(10^{6} / \mathrm{L}\right)$ & 673 & 37 & 102 & 172 & 5327 & 1760 & 1886 \\
\hline Monocyte percentage $(\%)$ & 5 & 74 & 60 & 40 & 18 & 5 & 2 \\
\hline $\begin{array}{l}\text { Multinucleated cell } \\
\text { percentage }(\%)\end{array}$ & 95 & 26 & 40 & 60 & 82 & 95 & 98 \\
\hline SARS-CoV-2(RNA) & - & - & - & - & - & - & - \\
\hline
\end{tabular}


L, among which polykaryotic cells accounted for $60 \%$. The cause of fever was considered to be intracranial infection; gram-negative bacilli were more likely. Continue treatment against infection.

On March 7, the patient developed high fever, up to $41^{\circ} \mathrm{C}$, and the body temperature was not easy to control. The cerebrospinal fluid was yellow with clots, protein $4.29 \mathrm{~g} / \mathrm{L}$, glucose $0.4 \mathrm{mmol} / \mathrm{L}$, and white blood cells increased to $5327 \times$ $10^{6} \mathrm{G} / \mathrm{L}$, among which polykaryotic cells accounted for $82 \%$. The patient had a clear history of cerebral hemorrhage, pus was drained from the lumbar cistern drainage tube, and cerebrospinal fluid analysis indicated pyogenic infection, especially the drug-resistant gram-negative bacilli. Clinical pharmacists suggested meropenem combined with rifampicin to fight the infection. Yeast was cultured in the patient's sputum, and the possibility of fungal infection could not be ruled out, and voriconazole antifungal treatment was added. In addition, central fever needs to be considered.

On March 8, the culture results of cerebrospinal fluid showed acinetobacter baumannii. Considering opportunistic pathogenic infections, after in-hospital consultations, tigecycline and cefoperazone and sulbactam were given intrathecally by intrathecal injection, and oral minocycline. Continue to use voriconazole against fungus.

On March 10, dark red substances were drained from the gastric canal and black stools were observed in the patient, considering gastrointestinal bleeding, hemostatic drugs were administered, low-temperature noradrenaline dilution solution and low-temperature thrombin solution were injected into the gastric canal respectively, but the effect was poor.

On March 11, the patient still had gastrointestinal bleeding, and his body temperature was $37.5^{\circ} \mathrm{C}$. Brain CT examination showed that the range of patchy high-density lesions was significantly smaller than that before (March 2, 2020), the patchy low-density shadows were increased, and the left brain tissue was significantly swollen; the left ventricle was compressed and deformed, and the scalp soft tissue was swollen. Reexamination of the chest $\mathrm{CT}$ showed that there were patches and strip-like high-density shadows on the back of both lungs, with a blurred state and some consolidation, which was more than before (March 2, 2020). In order to alleviate the condition and supplement the patient's coagulation factors, $200 \mathrm{ml}$ of type A Rh-positive plasma was given after the consent of the patient's family. The patient's gastrointestinal bleeding stopped and the temperature gradually returned to normal. The re-examination of SARS-CoV-2 nucleic acid test was still negative on March 19, 2020.

\section{Discussion}

SARS-CoV-2 can invade the cardio-cerebrovascular system and damage the nervous system, which has been reported in several articles [8-14]. Angiotensin converting enzyme 2(ACE2) is highly expressed in human tissues such as the vascular endothelial cells, heart, liver, digestive tract, and kidneys $[15,16]$. Studies have found that ACE2 is an important target for SARS-CoV-2 infection in humans. After infection, ACE2 levels are reduced or even absent. Therefore, it was learned that all tissues and organs expressing ACE2 may be the battlefield between SARS-CoV-2 and immune cells, eventually leading to multi-organ failure and life-threatening [17].

After infection with SARS-CoV-2, this patient did not first appear classic respiratory clinical symptoms, but presented with cerebral hemorrhage. Whether this is a coincidence or an inevitable correlation, the pathophysiological mechanism requires further study. As we all know, cerebral hemorrhage refers to spontaneous hemorrhage in nontraumatic brain parenchyma. Common causes are hypertension, cerebral arteriosclerosis, intracranial vascular malformation, cerebral amyloid vascular disease, hematopathy, vasculitis, etc. The vast majority of cerebral hemorrhage is manifested as small artery rupture caused by hypertension, which can cause extremely serious symptoms within a short period of time, and even affect patients' basic physiological activities such as breathing and heartbeat, resulting in death. It is a common acute cerebrovascular disease in middleaged and elderly people, with a high fatality and disability rate [18]. The patient had no history of hypertension, sudden cerebral hemorrhage, and no vascular abnormalities such as aneurysms during the operation. Is the occurrence of cerebral hemorrhage in patients related to the consumption of ACE2 by SARS-CoV-2?

The patient had no exact close contact history, and chest CT did not have the typical COVID-19 features, which may be related to the mask of combined hypostatic pneumonia. However, in combination with the patient's high fever, lymphocytopenia, increased neutrophils, and poor antibacterial treatment effect, the clinical manifestations conform to the COVID-19 characteristics, and nucleic acid detection is required. As is known to all, nucleic acid detection is the gold standard for the diagnosis of SARS-CoV-2 infection, but the first test result is negative, which is related to the low positive rate of SARS-CoV-2 detection using fluorescent quantitative RT-PCR as the detection method, currently only 30 to $50 \%$, leading to an extremely high false negative rate [19]. Because the test results are affected by a variety of factors, including: in vivo viral load, whether the specimen is qualified, whether the transport conditions meet the standards, whether the specimen is contaminated by RNA enzymes resulting in nucleic acid degradation, etc. The patient obtained a positive result on the second test and was eventually diagnosed with COVID19. Therefore, we believe that the standardized collection and transportation of specimens and repeated testing are conducive to the improvement of positive rate, early diagnosis and early treatment. 
Studies have found that the susceptibility of SARS-CoV-2 is related to blood type. Among 1775 COVID-19 patients at Wuhan Jinyintan Hospital, the analysis results showed that the proportion of $\mathrm{A}, \mathrm{B}, \mathrm{AB}$, and $\mathrm{O}$ accounted for $37.75 \%$, $26.42 \%, 10.03 \%$, and $25.80 \%$. Compared with blood types $\mathrm{O}, \mathrm{B}$, and $\mathrm{AB}$, the risk of COVID-19 infection in blood group A was significantly increased (OR was $1.20 ; 95 \%$ confidence interval CI was $1.02-1.43, P=0.02$ ) [20]. The patient had blood type A and was susceptible. Nevertheless, are patients with type A more likely to develop cerebrovascular accidents after SARS-CoV-2 infection? This requires large sample studies.

When the patient was critically ill, $150 \mathrm{ml}$ of type A Rh-positive COVID-19 convalescent plasma was injected. After treatment, the nucleic acid turned negative after multiple re-examinations, indicating that the treatment was effective. From the perspective of clinical pathology, IgM antibodies produced after human infection with the virus gradually decline after reaching the peak in the acute infection period, and usually persist for $8-12$ weeks or longer. Most COVID-19 patients will produce specific antibodies against SARS-CoV-2 in their bodies. After treatment and rehabilitation, IgG antibodies are of therapeutic value are found in the plasma. These specific antibodies are injected into the body and neutralized with SARS-CoV-2 to achieve the purpose of virus suppression and elimination. However, in fact, in addition to the "antibody pathway", the specific immunoglobulin in the plasma of the convalescent patients can also play a therapeutic role, and even the plasma itself has a certain role [21, 22].

It is important to note that the plasma composition is complex and there is uncertainty as to whether it will cause other problems. In clinical use, it is necessary to grasp the indications, contraindications, and unsuitable conditions. Indications for clinical use of recovered plasma, nucleic acid test positive and rapid progression, critical COVID-19 patients, should be used as soon as possible; in principle, the course of disease does not exceed 3 weeks. Contraindications: terminal stage of critical illness, multiple organ failure cannot be reversed; non-neutralizing treatment of SARS-CoV-2; comprehensive assessment by the clinician that there are other unsuitable infusion situations.

After SARS-CoV-2 infection, most patients were cured, but many patients progressed to severe illness and eventually died. Respiratory failure is the main cause of death, followed by sepsis (multiple organ failure), heart failure, bleeding, and kidney failure. The affected organs mainly include the respiratory system, cardiovascular system, coagulation function, and the liver and the kidney. In many cases, multiple organ functions are impaired. Through the study of death cases, it was found that in addition to directly attacking human tissues and organs, SARS-CoV-2 caused a cytokine storm by stimulating the human immune system. Many patients with severe COVID-19 will develop cytokine storm, which is an over- immune response of the human body. The main reason is the over-activation of non-specific immune cells, which release a large number of pro-inflammatory factors, such as interleukin-1, interleukin-6, and tumor necrosis factor. [23]. Therefore, it is suggested that clinicians should take a twopronged approach to severely ill patients with COVID-19, in addition to paying special attention to the protection of organ function, but also need to strengthen the control of cytokine storm.

The patient presented with cerebral hemorrhage at the time of onset, and soon developed high fever, dyspnea, and difficulty in maintaining blood oxygen saturation, requiring ventilator-assisted breathing. The early lung CT did not have the typical manifestations of COVID-19. Although the symptoms did not improve after the antibacterial treatment, the lung $\mathrm{CT}$ gradually showed the image of COVID-19.

The patient's cerebral hemorrhage was treated early and the hematoma was absorbed. When diagnosed, after antiviral and plasma treatment, the patient's symptoms improved, and the cerebrospinal fluid improved, but later swelling of the brain tissue and purulent cerebrospinal fluid occurred. This was considered because SARS-CoV-2 infection damaged the immune system [24]. In the late stage, the immune system was weakened, opportunistic pathogens invaded again, and fungal infection occurred, making the infection difficult to control. In addition, the patient also developed gastrointestinal bleeding, which was difficult to control with ordinary hemostatic drugs. Finally, the patient's symptoms gradually stabilized by re-infusion of plasma.

Through this case, we have gained experience: COVID-19 is highly contagious, and although there is no clear history of close contact, infection is still possible. Early detection of highly suspected cases helps early treatment. SARS-CoV-2 can damage the neurovascular system and cause bleeding, and it can be followed by multiple system failure. Early treatment is beneficial to the prognosis. SARS-CoV-2 can lead to a weakened immune system and secondary opportunistic pathogenic infections. It is necessary to focus on improving immunity as soon as possible.

The shortcomings of this article: lack of magnetic resonance data, lack of pathological tissue data, and lack of substantial evidence.

Acknowledgments The authors thank all the medical staff who provided the patient with careful treatment and care.

\section{Compliance with Ethical Standards}

Competing Interests The authors declare that they have no competing interests.

Ethical Approval All relevant ethical guidelines have been followed. 


\section{References}

1. Wu F, Zhao S, Yu B, Chen YM, Wang W, Song ZG, et al. A new coronavirus associated with human respiratory disease in China. Nature. 2020;579(7798):265-9.

2. Guan W, Ni Z, Hu Y, Liang WH, Ou CQ, He JX, et al. Clinical characteristics of coronavirus disease 2019 in China. N Engl J Med. 2020;382:1708-20. https://doi.org/10.1056/NEJMoa2002032.

3. Huang C, Wang Y, Li X, Ren L, Zhao J, Hu Y, et al. Clinical features of patients infected with 2019 novel coronavirus in Wuhan. China The Lancet. 2020;395(10223):497-506.

4. Wu Z, McGoogan JM. Characteristics of and important lessons from the coronavirus disease 2019 (COVID-19) outbreak in China: summary of a report of 72314 cases from the Chinese Center for Disease Control and Prevention. Jama. 2020;323:1239. https://doi.org/10.1001/jama.2020.2648.

5. Zhou F, Yu T, Du R, et al. Clinical course and risk factors for mortality of adult inpatients with COVID-19 in Wuhan, China: a retrospective cohort study. Lancet. 2020;395:1054-62. https://doi. org/10.1016/S0140-6736(20)30566-3.

6. Li B, Yang J, Zhao F, Zhi L, Wang X, Liu L, et al. Prevalence and impact of cardiovascular metabolic diseases on COVID-19 in China. Clin Res Cardiol. 2020;109:1-8. https://doi.org/10.1007/ s00392-020-01626-9.

7. Naicker S, Yang CW, Hwang SJ, Liu BC, Chen JH, Jha V. The novel coronavirus 2019 epidemic and kidneys. Kidney Int. 2020;97:824-8. https://doi.org/10.1016/j.kint.2020.03.001.

8. Li YC, Bai WZ, Hashikawa T. The neuroinvasive potential of SARS-CoV2 may be at least partially responsible for the respiratory failure of COVID-19 patients. J Med Virol. 2020;92:552-5. https:// doi.org/10.1002/jmv.25728.

9. Mao L, Wang M, Chen S, et al. Neurological manifestations of hospitalized patients with COVID-19 in Wuhan, China: a retrospective case series study. Lancet. 2020; https://ssrn.com/ abstract $=3544840$

10. Ye M, Ren Y, Lv T. Encephalitis as a clinical manifestation of COVID-19. Brain Behav Immun. 2020 Apr 10. pii: S08891591(20)30465-7. https://doi.org/10.1016/j.bbi.2020.04.017. [Epub ahead of print].

11. Butowt R, Bilinska K. SARS-CoV-2: olfaction, brain infection, and the urgent need for clinical samples allowing earlier virus detection. ACS Chem Neurosci. 2020;11:1200-3. https://doi.org/10.1021/ acschemneuro.0c00172. [Epub ahead of print].

12. Moriguchi T, Harii N, Goto J, et al. A first case of meningitis/ encephalitis associated with SARS-Coronavirus-2. Int J Infect Dis. 2020. pii: S1201-9712(20)30195-8. https://doi.org/10.1016/ j.ijid.2020.03.062. [Epub ahead of print].
13. Li B, Yang J, Zhao F, Zhi L, Wang X, Liu L, et al. Prevalence and impact of cardiovascular metabolic diseases on COVID-19 in China. Clin Res Cardiol. 2020;109:531-8. https://doi.org/10. 1007/s00392-020-01626-9.

14. Bordi L, Nicastri E, Scorzolini L, et al. Differential diagnosis of illness in patients under investigation for the novel coronavirus (SARS-CoV-2), Italy, February 2020. Eurosurveillance. 2020;25(8). https://doi.org/10.2807/1560-7917.ES.2020.25.8. 2000170.

15. Alenina N, Bader M. ACE2 in brain physiology and pathophysiology: evidence from transgenic animal models. Neurochem Res. 2019;44(6):1323-9.

16. Wu X, Zhu B, Zou S, Shi J. The association between ACE2 gene polymorphism and the stroke recurrence in Chinese population. $\mathrm{J}$ Stroke Cerebrovasc Dis. 2018;27(10):2770-80.

17. Yan R, Zhang Y, Li Y, Xia L, Guo Y, Zhou Q. Structural basis for the recognition of the SARS-CoV-2 by full-length human ACE2. Science. 2020;367:1444-8. https://doi.org/10.1126/science. abb2762.

18. Toyoda K, Koga M, Yamamoto H, Foster L, Palesch YY, Wang Y, et al. Clinical outcomes depending on acute blood pressure after cerebral hemorrhage. Ann Neurol. 2019;85(1):105-13.

19. Wang Y, Kang H, Liu X, Tong Z. Combination of RT-qPCR testing and clinical features for diagnosis of COVID-19 facilitates management of SARS-CoV-2 outbreak. J Med Virol. 2020;92:538-9. https://doi.org/10.1002/jmv.25721.

20. Zhao J, Yang Y, Huang HP, et al. Relationship between the ABO blood group and the COVID-19 susceptibility. medRxiv. 2020 . https://doi.org/10.1101/2020.03.11.20031096.

21. Hoffmann M, Kleine-Weber H, Schroeder S, Krüger N, Herrler T, Erichsen S, et al. SARS-CoV-2 cell entry depends on ACE2 and TMPRSS2 and is blocked by a clinically proven protease inhibitor. Cell. 2020;181:271-280.e8. https://doi.org/10.1016/j.cell.2020.02. 052.

22. Chen L, Xiong J, Bao L, Shi Y. Convalescent plasma as a potential therapy for COVID-19. Lancet Infect Dis. 2020;20:398-400. https://doi.org/10.1016/S1473-3099(20)30141-9.

23. Zhang B, Zhou X, Qiu Y, et al. Clinical characteristics of 82 death cases with COVID-19. medRxiv. 2020. https://doi.org/10.1101/ 2020.02.26.20028191.

24. Huang L, Shi Y, Gong B, et al. Blood single cell immune profiling reveals the interferon-MAPK pathway mediated adaptive immune response for COVID-19. medRxiv. 2020. https://doi.org/10.1101/ 2020.03.15.20033472.

Publisher's Note Springer Nature remains neutral with regard to jurisdictional claims in published maps and institutional affiliations. 